\title{
Different patterns of extracellular matrix protein expression in the convexity and the concavity of the dilated aorta with bicuspid aortic valve: Preliminary results
}

Maurizio Cotrufo, MD, ${ }^{a}$ Alessandro Della Corte, MD, ${ }^{a}$ Luca S. De Santo, MD, ${ }^{a}$ Cesare Quarto, MD, ${ }^{a}$ Marisa De Feo, MD, ${ }^{a}$ Gianpaolo Romano, MD, ${ }^{\mathrm{b}}$ Cristiano Amarelli, MD, Michelangelo Scardone, MD, ${ }^{\mathrm{b}}$ Franca Di Meglio, MD, ${ }^{\mathrm{c}}$

Germano Guerra, MD, ${ }^{\mathrm{c}}$ Maria Scarano, MD, ${ }^{\mathrm{c}}$ Serena Vitale, MD, ${ }^{\mathrm{c}}$ Clotilde Castaldo, MD, ${ }^{\mathrm{c}}$ and Stefania Montagnani, $\mathrm{MD}^{\mathrm{c}}$

Additional material is available online. 角
From the Department of Cardiothoracic and Respiratory Sciences, Second University of Naples, ${ }^{a}$ the Department of Cardiovascular Surgery and Transplants, V. Monaldi Hospital, Naples, ${ }^{\mathrm{b}}$ and the Department of Biomorphological and Functional Sciences, Federico II University, Secondo Policlinico, Naples, Italy.

Received for publication Oct 29, 2004; revisions received Jan 3, 2005; accepted for publication Jan 18, 2005

Address for reprints: Alessandro Della Corte, MD, Via A. Modigliani 64, 81031, Aversa CE, Italy (E-mail: aledellacorte@ libero.it).

J Thorac Cardiovasc Surg 2005;130:504-11 $0022-5223 / \$ 30.00$

Copyright (C) 2005 by The American Association for Thoracic Surgery

doi:10.1016/j.jtcvs.2005.01.016
Objective: This study aimed to assess extracellular matrix protein expression patterns at the convexity (right anterolateral wall) and the concavity of the dilated ascending aorta in patients with bicuspid aortic valve disease.

Methods: Aortic wall specimens were retrieved from the convexity and the concavity in 27 bicuspid aortic valve patients (12 with stenosis and 15 with regurgitation) and 6 heart donors (controls). Morphometry, immunohistochemistry, Western blot, and polymerase chain reaction were performed, focusing on matrix proteins involved in vascular remodeling.

Results: Type I and III collagens were significantly decreased in bicuspid-associated dilated aortas versus controls $(P<.001)$, particularly at the convexity $(P<.05$ vs concavity). Expression of messenger RNA for collagens was lower than normal only in the regurgitant subgroup. At immunohistochemistry, proteins whose overproduction has been demonstrated in response to abnormal wall stress, such as tenascin and fibronectin, were more expressed in the convexity than in the concavity, especially in the stenosis subgroup. Tenascin, which is produced by smooth muscle cells in the synthetic phenotype, was nearly undetectable in controls. Fewer smooth muscle cells (stenosis, $P=.017$; regurgitation, $P=.008$ ) and more severe elastic fiber fragmentation $(P=.029$ and $P<.001)$ were observed in the convexity versus the concavity.

Conclusions: In bicuspid-associated aortic dilations, an asymmetric pattern of matrix protein expression was found that was consistent with the asymmetry in wall-stress distribution reported previously. Differences exist between patients with stenosis and those with regurgitation in terms of protein expression and content in the aortic wall. Further studies could clarify the relations between these findings and the pathogenesis of aortic dilatation in bicuspid aortic valve patients.

$\mathrm{T}$ he pathogenesis of medial degeneration of the aorta in bicuspid aortic valve (BAV) disease is undefined. Recently, an abnormality in the process of extracellular matrix (ECM) remodeling (inadequate synthesis, degradation, and/or transport of ECM proteins) in the aortic wall has been advocated as a possible mechanism. ${ }^{1,2}$ The association of aortic wall abnormalities with BAV may derive from an inherited developmental defect of neural crest cells, which contribute to both aortic valve and wall embryogenesis, ${ }^{3,4}$ although a specific responsible gene has never been identified. Conversely, some authors have argued that the aortic wall pathology could be mainly secondary to abnormal postvalvular hemodynamics, and wall-stress overload at the right anterolateral aspect (convexity) has been demonstrated. ${ }^{5}$ Flow characteristics are known to influence the morphology and even the microstructure of the vessel wall. ${ }^{6}$ 
This study was undertaken to assess the patterns of expression of histologic and morphometric features and of ECM proteins in aneurysmal ascending aortas of patients with BAV. Among ECM components involved in the processes of tissue remodeling, collagen, fibronectin, laminin, and tenascin were object of analysis in this study. Our interest was focused on type I and III collagen, ${ }^{7}$ belonging to the class of fibrillar collagens, and type IV collagen, which is a nonfibrillar type and participates in basement membrane constitution. Fibronectin in the human aorta is abundantly represented in the basal lamina-like layers that connect the cells to each other and to the oxytalan fibers. ${ }^{8}$ The laminins are glycoproteins localized in the basal lamina. They regulate cellular differentiation, proliferation, and migration. Among the 5 types of tenascin described so far, tenascin $\mathrm{C}$ plays crucial roles in embryonic development and tissue remodeling. ${ }^{9}$

\section{Methods}

\section{Patients and Retrieval Methods}

From 2000 to 2003, a total of 143 patients underwent surgery for ascending aorta or aortic root dilatation at the authors' institution. Only patients with a clearly congenital bicuspid valve, as assessed by both patient history and intraoperative examination and confirmed at pathology, were included in the study. The patients whose aortic valves became bicuspid during their lifetime because of either rheumatic or degenerative leaflet fusion were excluded, as were those with atherosclerosis of the ascending aorta, aortitis, infective endocarditis, and primary connective tissue disorders, eg, Marfan syndrome. To reduce the heterogeneity of local hemodynamic conditions, BAV patients with hypertension and those with mixed valve disease (combined stenosis and regurgitation) were not included. Thus, 27 patients constituted the study sample, all with BAV disease and proximal aortic dilatation (Table E1): 12 had aortic valve pure stenosis (mean age, $59 \pm 12.1$ years), and 15 had isolated aortic valve regurgitation (mean age, $56.3 \pm 9.2$ years). During the operation, the aortic dilatation appeared asymmetric in all cases: 2 samples of aortic wall were harvested from each patient at the aortic convexity (right anterolateral aspect, above the noncoronary sinus) and concavity (opposite wall), $2 \mathrm{~cm}$ beyond the sinotubular junction. Samples were sectioned into 4 specimens each: 1 was used for histology and morphometry, 1 for Western blot analysis, and 1 for reverse transcription-polymerase chain reaction (RT-PCR), and the last specimen was paraffinembedded for immunohistochemistry. To be able to define whether each studied protein was increased or reduced in the diseased aorta, we chose as a control the normal aorta. Therefore, aortic wall specimens were retrieved during multiorgan harvesting from 6 normotensive heart donors who had no aneurysmal or atherosclerotic disease of the aorta or BAV (mean age, $33 \pm 8$ years). Sites of retrieval in control subjects were the same as described previously. The study was approved by our institution's ethics committee.

\section{Echocardiography}

Two-dimensional transthoracic and transesophageal echocardiography was performed with an Acuson Sequoia C256 machine
(Mountain View, Calif) with a multifrequency 3V2C sectorial transducer. Aortic valve morphology was evaluated in parasternal long-axis and short-axis views: a BAV was diagnosed when only 2 cusps were clearly identified in systole and diastole in short-axis view.

\section{Western Blot}

Samples were washed in phosphate-buffered saline (PBS) and then lysed by lysis buffer (1\% Triton X-100 in PBS) containing protease inhibitors for 30 minutes at $4^{\circ} \mathrm{C}$. Lysates were centrifuged at $10,000 \mathrm{rpm}$ for 10 minutes at $4^{\circ} \mathrm{C}$; protein concentration was determined by protein assay (Bio-Rad, Richmond, Colo). For each sample, the same amount of total proteins was added with Laemmli sample buffer and separated by electrophoresis in $30 \%$ sodium dodecyl sulfate-polyacrylamide gel. Gels were then electroblotted on polyvinylidene difluoride filters (Millipore, Bedford, Mass); membranes were blocked by $6 \%$ fat-free dry milk for 1 hour at room temperature. After 3 washes in washing solution, membranes were incubated overnight at $4{ }^{\circ} \mathrm{C}$ with antibodies against laminin; tenascin; type I, III, and IV collagens (Sigma, St Louis, Mo); or anti-fibronectin (Chemicon Int, Temecula, Calif). After washing, membranes were incubated for 45 minutes at room temperature with horseradish peroxidase-conjugated goat secondary antibodies (Bio-Rad). After further washing, membranes were developed in luminol substrate and exposed to film (ECL for laminin and fibronectin; ECLplus for tenascin; Amersham Biosciences, Little Chalfont, Buckinghamshire, United Kingdom). Computer-acquired images were quantified by using ImageQuant software (Amersham Biosciences).

\section{Reverse Transcription-Polymerase Chain Reaction}

RT-PCR was used to analyze target gene expression in this study. Total RNA was isolated by lysing the frozen aortic vessel tissue samples (150-300 mg) in Trizol solution (GIBCO BRL, Life Technologies, Rockville, Md) according to the supplier's protocol. RNA was precipitated and quantified by spectroscopy. Two micrograms of total RNA from each sample was reverse-transcribed by using the First-Strand cDNA Synthesis Kit (Amersham Pharmacia Biotech, Arlington Heights, Ill) according to the protocol supplied by the manufacturer. The random hexamer primers provided in the kit were used. The same complementary DNA product obtained from each sample was used for subsequent PCR amplification with the primer sets prepared for the target gene and the glyceraldehyde phosphate dehydrogenase (GAPDH) housekeeping gene. See Appendix E1 (online only) for primer sequences. The amplified products (12 $\mu \mathrm{L}$ of each sample) were analyzed by electrophoresis in a $2 \%$ agarose gel containing ethidium bromide, followed by photography under ultraviolet illumination. The levels of ECM protein messenger RNA (mRNA) were estimated by densitometric scanning and normalized against GAPDH loading controls. Densitometric analyses of the PCR products were performed with ImageJ software version 1.29 (developed by Wayne Rasband), available online at http://rsb.info.nih.gov/ij/. All PCR products were purified by using the QIAquick PCR purification kit (Qiagen, Santa Clarita, Calif), and their identities were verified by automated DNA forward and reverse sequencing with dideoxy terminator reaction chemistry for sequence analysis on the Applied Biosystems (Foster City, Calif) model 373A DNA sequencer. 
TABLE 1. Western blot results

\begin{tabular}{|c|c|c|c|c|}
\hline Variable & Normal & Regurgitant BAV & Stenotic BAV & $P$ value* \\
\hline \multicolumn{5}{|l|}{ Collagen I } \\
\hline Convexity & $52.3 \pm 2.1$ & $10.7 \pm 2.1 \dagger$ & $20.4 \pm 1.3 \dagger$ & $<.001$ \\
\hline Concavity & $51.0 \pm 2.0$ & $15.2 \pm 2.1$ & $25.6 \pm 1.6$ & $<.001$ \\
\hline \multicolumn{5}{|l|}{ Collagen III } \\
\hline Convexity & $25.8 \pm 3.7$ & $15.1 \pm 2.5 \dagger$ & $18.6 \pm 1.4 \dagger$ & .003 \\
\hline Concavity & $26.1 \pm 2.6$ & $18.1 \pm 1.6$ & $24.0 \pm 1.5$ & $<.001$ \\
\hline \multicolumn{5}{|l|}{ Collagen IV } \\
\hline Convexity & $15.1 \pm 1.2$ & $25.5 \pm 1.4 \dagger$ & $46.3 \pm 1.6 \dagger$ & $<.001$ \\
\hline Concavity & $14.1 \pm 2.6$ & $35.4 \pm 1.8$ & $45.5 \pm 2.1$ & $<.001$ \\
\hline \multicolumn{5}{|l|}{ Fibronectin } \\
\hline Convexity & $49.8 \pm 2.4$ & $52.5 \pm 2.3$ & $54.0 \pm 1.8 \dagger$ & .22 \\
\hline Concavity & $48.5 \pm 2.2$ & $51.2 \pm 4.5$ & $44.1 \pm 1.2$ & $<.001$ \\
\hline \multicolumn{5}{|l|}{ Laminin } \\
\hline Convexity & $38.1 \pm 0.7 \dagger$ & $45.2 \pm 3.8 \dagger$ & $33.5 \pm 3.2 \dagger$ & $<.001$ \\
\hline Concavity & $51.8 \pm 0.5$ & $50.3 \pm 4.1$ & $41.7 \pm 4.2$ & $<.001$ \\
\hline
\end{tabular}

All differences (Bonferroni correction) between the amounts of each protein (expressed in nanomoles) in each patient group and the corresponding amounts in controls were significant $(P \leq .001$ for collagens; $P<.05$ for laminin and fibronectin), except for collagen III in stenotic BAV versus normal (concavity: $P=.10$ ) and for fibronectin (concavity: $P=.09$ ) and laminin (concavity: $P=.40$ ) in regurgitant BAV versus normal. $B A V$, Bicuspid aortic valve. *Regurgitant BAV versus stenotic BAV. $\uparrow P<.05$, convexity versus concavity (paired $t$ test).

\section{Immunohistochemistry}

Specimens were fixed in buffered $10 \%$ formalin, embedded in paraffin, and sectioned. Serial $4-\mu \mathrm{m}$-thick sections of aortic specimens were deparaffinized; covered with primary monoclonal antibodies against types I, II, and IV collagen, tenascin, and laminin or polyclonal antibody for fibronectin (Sigma) and type III collagen (Santa Cruz Biotechnology Inc, Santa Cruz, Calif); and incubated in a moist chamber for 1 hour at $37^{\circ} \mathrm{C}$. After some washings in PBS, they were covered with fluoresceinated secondary antibodies (Sigma) and submitted to the same treatment. Nuclei were stained with propidium iodide, and then sections were mounted in Vectashield (Vector Laboratories, Burlingame, Calif) and observed with a Leica DMLB fluorescence microscope (Leica Microsystems, Inc, Bannockburn, Ill), and a Zeiss LSM510 confocal microscope (Karl Zeiss International, Jena, Germany). Samples were evaluated by 3 independent observers by using a 4-point scale to quantify staining, from none $(-)$ to greatest $(+++) .{ }^{10}$

\section{Histology and Morphometry}

Specimens were prepared with hematoxylin-eosin, periodic acidSchiff, Weigert-van Gieson stain, Alcian-periodic acid-Schiff, Alcian-Weigert stain for elastic fibers, and von Kossa stain. Histologic diagnosis was expressed through the grading system suggested by Schlatmann and Becker ${ }^{11}$ for medial degeneration. Morphometric analysis was performed by using 2 programs (RM 2100 or RM 5200) of a computer-assisted image-analysis system (VIDAS Kontron Elektronik; Zeiss). For each specimen, 10 microscopic fields $(620 \times)$ were randomly selected; the number of elastic fibers, total smooth muscle cells (SMCs), and normal SMCs alone (excluding those with signs of cell degeneration) was counted; and mean values were computed. The length of elastic fibers, considering their angulation, and their density per field were also measured.

\section{Statistical Analysis}

SPSS software (version 10.1; SPSS Inc, Chicago, Ill) was used for statistical analysis. Continuous variables were summarized as mean \pm standard deviation. Morphometry, Western blot, and PCR findings were compared between the convexity and the concavity within each patient group through paired $t$ tests. Differences among the 3 groups of specimens (control, regurgitant, and stenotic) were analyzed by 1-way analysis of variance with Bonferroni correction for post hoc comparisons.

\section{Results \\ Echocardiography}

Echocardiography showed that the mean ascending aortic diameter was $5.5 \pm 1.01 \mathrm{~cm}$ in the patients with aortic regurgitation and $5.7 \pm 1.02 \mathrm{~cm}$ in those with aortic stenosis $(P=.42)$. In all study patients, the greatest diameter was found beyond the sinotubular ridge, at the tubular tract level. Table E1 shows variables related to valve morphology and function. The pattern of cusp fusion was described at echocardiography and/or reported by the surgeon in the operative note. Twelve patients had a severe degree of valve regurgitation and 3 had a moderate degree; among those with aortic stenosis, it was severe in 10 and moderate in 2 .

\section{Western Blot}

A significantly decreased amount of type I and type III collagens was found in dilated aortas compared with controls, and those changes were more evident in specimens from the convexity of the aorta (Table 1). Type IV collagen was increased in dilated aortas: in stenotic BAVs, its amount was greater at the convexity $(P=.018)$, and in 


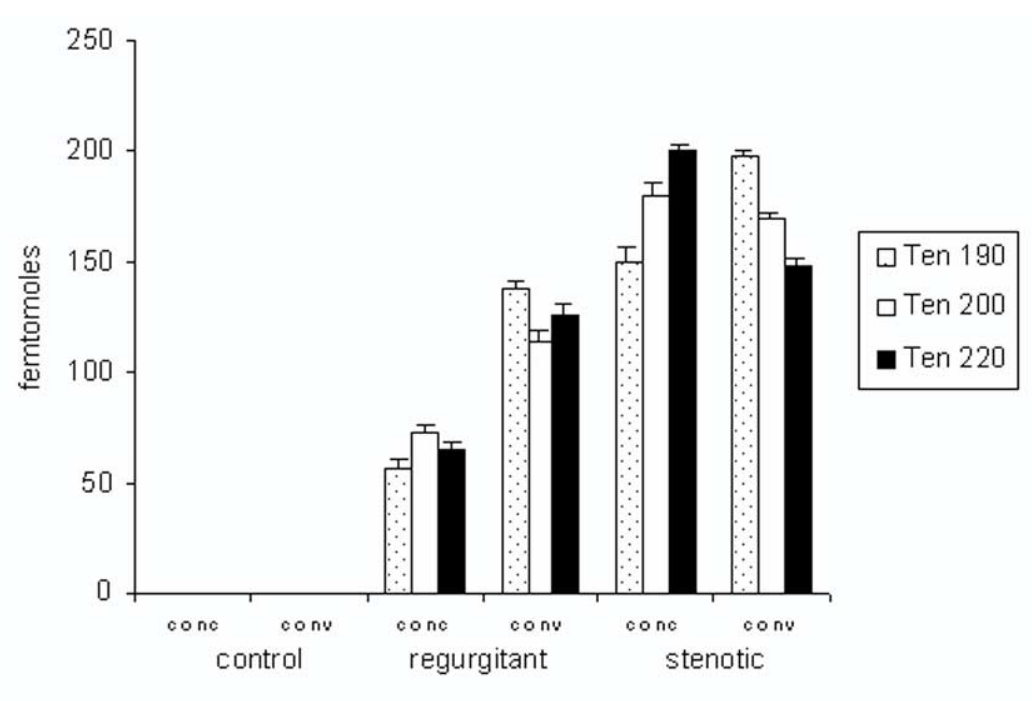

control RBAV SBAV

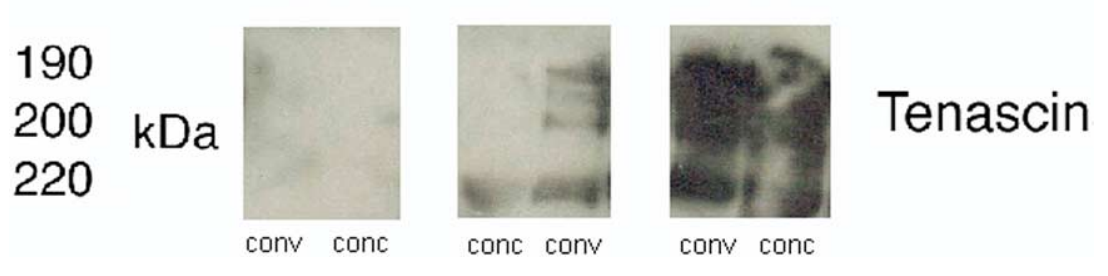

Figure 1. Tenascin expression evaluated by Western blot: comparison among controls, dilated aortas with regurgitant BAV, and dilated aortas with stenotic BAV. Ten, tenascin subunit (190, 200, and $220 \mathrm{kd}) ; R B A V$, regurgitant BAV; SBAV, stenotic BAV; conc, concavity; conv, convexity. regurgitant BAVs it was greater at the concavity $(P<.001)$. Fibronectin and laminin showed greater variability in dilated aortas. On average, patients had more fibronectin at the convexity than controls (Table 1). The laminin amount was significantly greater at the concavity than at the convexity in control aortas $(P<.001)$ : it appeared increased at the convexity in regurgitant BAVs, whereas in stenotic BAVs it was decreased. Tenascin $\mathrm{C}$ was not detectable in normal aortas, whereas its subunits were found in dilated aortas from BAV patients (Figure 1).

\section{Reverse Transcription-Polymerase Chain Reaction}

The mRNA for type I collagen (Figure 2) was reduced in dilated aortas with regurgitant $\operatorname{BAV}(P=.03)$; no significant differences were observed between convexity and concavity in all 3 groups. As to the mRNA for type III collagen, lower levels of expression at the concavity $(P=.01 \mathrm{vs}$ control concavity) were found in regurgitant BAVs (without a significant difference between the 2 sites of retrieval), whereas stenotic BAVs showed nearly normal values. Collagen IV mRNA was equally expressed in normal concavity and convexity, and it was increased in regurgitant $(P=.22)$ and stenotic $(P=.07)$ BAVs. Figure 2 shows PCR results for fibronectin and laminin.

\section{Immunohistochemistry}

The mean thickness of the aortic wall, when measured in histologic sections, was $1.82 \pm 0.05 \mathrm{~mm}$ in concavity and $1.92 \pm 0.05 \mathrm{~mm}$ in convexity in controls, and it was, respectively, $1.313 \pm 0.06 \mathrm{~mm}$ and $1.24 \pm 0.04 \mathrm{~mm}$ in dilated aortas with BAV regurgitation and $1.12 \pm 0.04 \mathrm{~mm}$ and $0.97 \pm 0.06 \mathrm{~mm}$ with stenosis. The results of confocal immunofluorescence are summarized in Table 2.

\section{Histology and Morphometry}

At histology, medial degeneration was found in all specimens from patients. The mean degree of medial degeneration in patients with stenosis was $2.7 \pm 0.5$ in the convexity versus $1.8 \pm 0.6$ in the concavity $(P<.001)$; in patients with regurgitation, it was $2.5 \pm 0.5$ versus $1.7 \pm 0.6$, respectively $(P<.001)$. Morphometry results are displayed in Table 3. In both the regurgitant and the stenotic BAV groups, both total and normal SMC numbers were significantly reduced in the convexity when compared with the concavity. More elastic fibers in the convexity than in the concavity were observed in both groups. This was due to elastin fragmentation into shorter fibers; this increased their counted number but significantly reduced their length. 

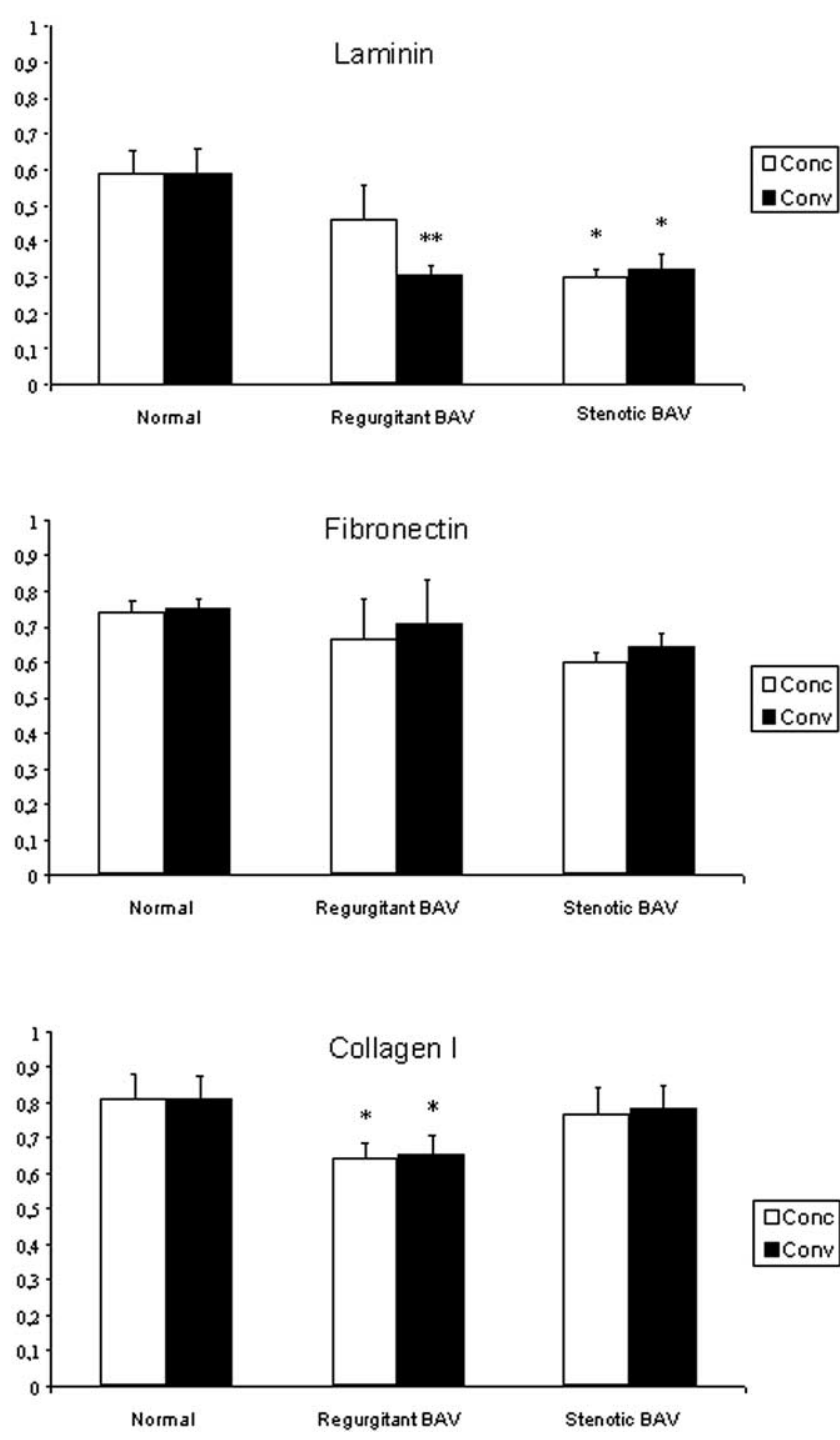

Figure 2. Messenger RNA amounts for laminin, fibronectin, and type I collagen as estimated by PCR. Regarding fibronectin, the great variability between patients observed on Western blot was also confirmed at PCR, with a tendency toward higher levels at the convexity in all pathologic aortas. The mRNA for laminin was always decreased in pathologic samples when compared with normal ones: in regurgitant BAVs, its expression was significantly lower at the convexity than at the concavity $(P=.005$; ${ }^{*} \boldsymbol{P}<.05$ for unpaired $\boldsymbol{t}$ test vs corresponding control specimens; ${ }^{* *} \boldsymbol{P}<.05$ both vs regurgitant concavity and vs control concavity). We tried to identify the oligonucleotidic sequence for tenascin $C$, but it was not possible to measure its mRNA. Conc, Concavity; Conv, convexity.

When histologic and morphometric findings were compared between stenotic and regurgitant BAVs, no significant differences emerged.

\section{Discussion}

In this pilot study, aortic wall changes in patients with BAV disease were found to be distributed in an asymmetric fashion and generally had more marked changes at the convex aspect. This finding could support the hypothesis of a role of mechanical stress in the development of BAVassociated dilations, because it is consistent with the evidence in the literature that the blood flow downstream from a BAV is eccentric, with consequent asymmetric aortic wall stress and a maximal burden at the convexity. ${ }^{5}$ Mostly, biomolecular data consisted with morphologic observations. The proven overall feasibility of the analysis encourages us to continue investigating this subject with the same methods. The criterion of separate analysis of hemodynamic subgroups (stenosis and regurgitation) was dictated by clinical experience which suggested that BAV patients are a heterogeneous group. ${ }^{1,12,13}$ Previous studies, which included different clinical phenotypes-such as normally functioning, stenotic, and regurgitant BAVs, as well as dilated and nondilated aortas-in the same series, led to discordant and irreproducible results. ${ }^{14-16}$

\section{Convexity Versus Concavity}

To our knowledge, so far only 1 study $^{2}$ has included proteins other than fibrillin among the ECM constituents investigated, but the site of tissue retrieval from the aorta (convexity or concavity) was not specified. Moreover, that was an in vitro investigation on cultured cells, and, therefore, it may have neglected the complex signal exchange between cells and matrix, ${ }^{17}$ as well as the interactions between medial components and mechanical stimuli that continuously occur in vivo. In experimental animals, ${ }^{18-20}$ mechanical factors such as the hypertensive stimulus and the high flow promoted the SMC change to the synthetic phenotype, apoptosis, fibronectin and tenascin overexpression, and release of matrix metalloproteinases. It is interesting to note that in this study, fibronectin and tenascin were more expressed in the aortic convexity-which is, according to previous studies, ${ }^{5,21,22}$ an area of stress concentration and flow turbulence-than in the concavity. Fibronectin is known to promote the modulation from the contractile to the synthetic phenotype, whereas laminin retains the cells in a contractile phenotype. ${ }^{17}$ SMCs in the synthetic state have been shown to produce tenascin both in vitro and in vivo. ${ }^{23}$ Tenascin was virtually absent in aortic wall specimens from healthy subjects in this study, and this is consistent with previous findings in normotensive animals. ${ }^{18}$ Conversely, its presence in specimens from BAV patients suggests SMC activation and a switch to the synthetic phenotype.

Type I and III collagens, the "scaffold" proteins mainly localized in fibers associated with the elastic lamellae, ${ }^{8}$ were more markedly decreased in the convex than in the concave aspect of diseased aortas when compared with normal aortas 
TABLE 2. Immunohistochemical examination of the aortic wall

\begin{tabular}{|c|c|c|c|c|c|c|}
\hline \multirow[b]{2}{*}{ Variable } & \multicolumn{3}{|c|}{ Collagen } & \multirow[b]{2}{*}{ Fibronectin } & \multirow[b]{2}{*}{ Tenascin } & \multirow[b]{2}{*}{ Laminin } \\
\hline & I & III & IV & & & \\
\hline \multicolumn{7}{|l|}{ Normal } \\
\hline Convexity & ++ & + & $+/-$ & + & $+1-$ & $+/++$ \\
\hline Concavity & ++ & $+/++$ & $+1-$ & + & $+1-$ & ++ \\
\hline \multicolumn{7}{|c|}{ Regurgitant BAV } \\
\hline Convexity & $+1-$ & $+1-$ & + & ++ & + & + \\
\hline Concavity & $+1-$ & $+1-$ & ++ & + & $+1-$ & ++ \\
\hline \multicolumn{7}{|l|}{ Stenotic BAV } \\
\hline Convexity & $+1-$ & $+1-$ & ++ & +++ & ++ & + \\
\hline Concavity & + & + & +++ & ++ & ++ & $+/-$ \\
\hline
\end{tabular}

In control subjects, the expression and the distribution of extracellular matrix (ECM) proteins were generally uniform between convexity and concavity: tenascin and type IV collagen were virtually absent. Type I and III collagens were less expressed in diseased aortas than in controls: an asymmetric distribution was found in the stenotic subgroup, with lower positivity in the convexity. Type IV collagen was increased in dilated aortas, particularly in stenotic bicuspid aortic valve (BAV) patients, with a greater amount at the concavity. Fibronectin was increased in dilated aortas, most evidently in the stenotic BAV subgroup, with always a more marked increase at the convexity. Immunostaining for tenascin was more evident in stenotic BAVs and only lightly in regurgitant BAVs at the convexity. Laminin was decreased in stenotic BAV concavity, whereas in regurgitant BAVs it was similar to controls.

(Figure 3). Collagens provide resistance to traction forces, and their asymmetric expression, along with the nonuniform severity of medial degeneration changes, is consistent with the common surgical finding of BAV-associated dilatations that predominantly involve the right anterolateral aspect, with the typical appearance of asymmetric dilatations, ${ }^{24}$ as were observed in this study.

\section{Stenotic Versus Regurgitant BAV}

Remarkable differences were observed in ECM components between the settings of regurgitant and stenotic valves. In particular, both type I collagen and its mRNA were strongly reduced in patients with BAV regurgitation when compared with healthy patients, whereas in the stenotic BAV patients, collagen was decreased, but its mRNA was not different from that of controls (and this could be considered a sign of a lack of adaptation to increased stress). Collagen reduction without a proportional reduction in its mRNA, however, suggests that collagen degradation by proteinases could prevail over collagen synthesis in the stenosis subgroup. Conversely, a decrease in the transcription of the protein was more evident in the regurgitation subgroup. Patients with ste- notic BAV also had less laminin and more fibronectin and tenascin than those with regurgitant BAV.

The jet stream through a stenotic valve causes greater radial stress on the ascending aorta, ${ }^{5,14}$ whereas the increased stroke volume that occurs with aortic regurgitation amplifies the ventricular traction on the aortic root that normally accompanies every heartbeat, thus augmenting longitudinal rather than radial stress, particularly at the right anterolateral wall. ${ }^{22}$ SMCs in the vessel wall could react differently to different types and degrees of mechanical stress and could favor, in turn, a predominant production of either ECM proteins or proteolytic enzymes. Otherwise, different possible gene defects might concomitantly condition both the type of dysfunction to which the valve is fated and the pattern of ECM protein production in the aortic wall. Aortic dilations associated with BAV stenosis and those with BAV regurgitation should be probably considered as different diseases that are possibly amenable to different therapeutic approaches.

\section{Study Limitations}

This study has the limitations of a pilot experience addressing such a complex issue as the microstructural changes in

TABLE 3. Morphometry results (mean number per field)

\begin{tabular}{|c|c|c|c|c|c|c|}
\hline \multirow[b]{2}{*}{ Variable } & \multicolumn{3}{|c|}{ Regurgitant BAVs } & \multicolumn{3}{|c|}{ Stenotic BAVs } \\
\hline & Convexity & Concavity & $P$ value* & Convexity & Concavity & $P$ value* \\
\hline Total SMCs & $274 \pm 132.8$ & $590 \pm 214.2$ & .001 & $356.7 \pm 36.9$ & $622.3 \pm 180.6$ & .014 \\
\hline Normal SMCs & $205 \pm 127.3$ & $562 \pm 73.9$ & .008 & $207 \pm 165.1$ & $437 \pm 73.9$ & .017 \\
\hline EF number & $949.3 \pm 513.9$ & $637.9 \pm 333.7$ & .165 & $937.5 \pm 188.4$ & $616.4 \pm 260.8$ & .002 \\
\hline Maximum EF length & $11.52 \pm 4.7$ & $13.01 \pm 2.5$ & .232 & $10.57 \pm 4.1$ & $13.62 \pm 1.9$ & .032 \\
\hline Minimum EF length & $0.41 \pm 0.1$ & $1.43 \pm 0.2$ & $<.001$ & $0.22 \pm 0.2$ & $1.46 \pm 0.6$ & .029 \\
\hline
\end{tabular}

$B A V$, Bicuspid aortic valve; SMCs, smooth muscle cells; $E F$, elastic fiber. *Concavity versus convexity. 

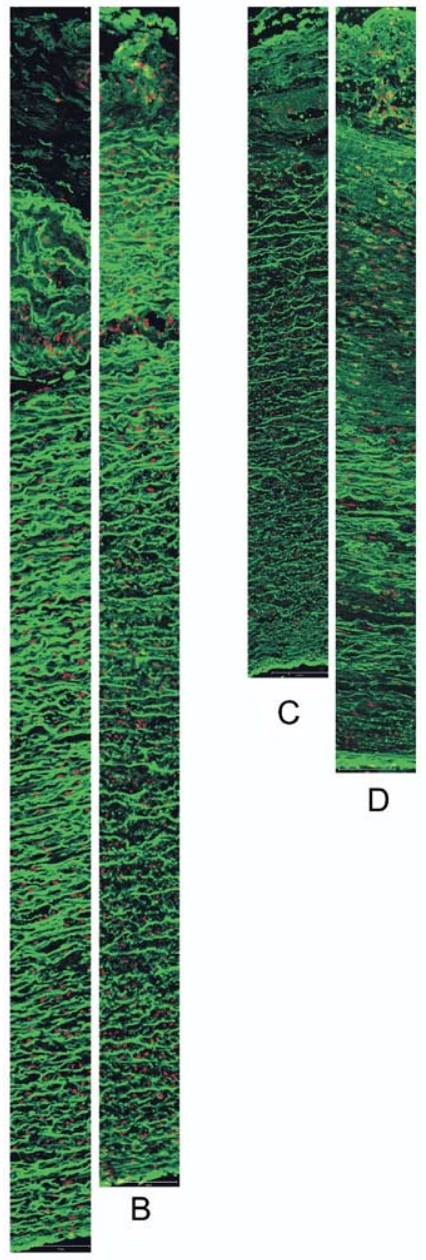

$\mathrm{D}$

A

Figure 3. Immunopositivity for type I collagen (in green; confocal microscopy) in the aortic wall (the intimal layer is above) in a patient with stenotic BAV. A, Healthy subject, concavity. B, Healthy subject, convexity. C, Regurgitant BAV, convexity. D, regurgitant BAV, concavity.

BAV-associated aortic dilatations. By now, the questions raised are perhaps more numerous than the answers provided. The asymmetric pattern of medial changes qualitatively and quantitatively coincides with the already-known wall-stress asymmetry in BAV disease; however, at this point, no clear causal mechanism can be demonstrated. Matching each patient's morphostructural data with the respective measurements of flow and stress characteristics (eg, through computed tomographic scan-derived patientspecific finite element models) will probably allow for a more direct and detailed description of the relations between wall stress and ECM changes. Finally, the normal setting (healthy valve, nondilated aorta) was chosen as the control group to recognize changes in patients. This was necessary for this first experience; however, further considerations could derive from comparing dilatations associated with BAV stenosis and regurgitation with those associated with tricuspid aortic valve stenosis and regurgitation of the same degree.

\section{Conclusions}

In conclusion, the novel finding of an asymmetric pattern of medial degeneration lesion distribution in BAV-associated aortic dilatations argues in favor of a possible central role of uneven wall stress in the pathogenesis of aortic dilatation. Among ECM proteins, fibronectin and tenascin were increased in the wall area of expected greater stress, whereas this relation was inverse for collagens I and III. Whether abnormal stress is the main causative factor or just interacts with an underlying genetic defect that might alter tissue adaptation to stress is still to be elucidated. Differences exist between patients with BAV stenosis and those with BAV regurgitation in terms of protein expression and content in the aneurysmal aortic wall; therefore, separate analysis of hemodynamic subgroups is advisable when this topic is addressed.

\section{References}

1. Fedak PW, de Sa MP, Verma S, Nili N, Kazemian P, Butany J, et al. Vascular matrix remodeling in patients with bicuspid aortic valve malformations: implications for aortic dilatation. J Thorac Cardiovasc Surg. 2003;126:797-806.

2. Nataatmadja M, West M, West J, Summers K, Walker P, Nagata M, et al. Abnormal extracellular matrix protein transport associated with increased apoptosis of vascular smooth muscle cells in marfan syndrome and bicuspid aortic valve thoracic aortic aneurysm. Circulation. 2003;108(suppl 1):II329-34.

3. Kappetein AP, Gittenberger-de Groot AC, Zwinderman AH, Rohmer J, Poelmann RE, Huysmans HA. The neural crest as a possible pathogenetic factor in coarctation of the aorta and bicuspid aortic valve. J Thorac Cardiovasc Surg. 1991;102:830-6.

4. Ward C. Clinical significance of the bicuspid aortic valve. Heart. 2000;83:81-5.

5. Robicsek F, Thubrikar MJ, Cook JW, Fowler B. The congenital bicuspid aortic valve: how does it function? Why does it fail? Ann Thorac Surg. 2004;77:177-85.

6. Lehoux S, Tedgui A. Cellular mechanics and gene expression in blood vessels. J Biomech. 2003;36:631-43.

7. Henkel W, Glanville RW. Covalent cross-linking between molecules of type I and type III collagen. The involvement of the $\mathrm{N}$-terminal, nonhelical region of the alpha1(I) and alpha1(III) chains in the formation of intermolecular cross-links. Eur J Biochem. 1982;122:205-13.

8. Dingemans KP, Teeling P, Lagendijk JH, Becker AE. Extracellular matrix of the human aortic media: an ultrastructural histochemical and immunohistochemical study of the adult aortic media. Anat Rec. 2000; 258:1-14.

9. Ikuta T, Ariga H, Matsumoto K. Extracellular matrix tenascin-X in combination with vascular endothelial growth factor B enhances endothelial cell proliferation. Genes Cells. 2000;5:913-27. 
10. Fox WM, Hameed A, Hutchins GM, Reitz BA, Baumgartner WA, Beschorner WE, et al. Perforin expression localizing cytotoxic lymphocytes in the intimas of coronary arteries with transplant-related arteriosclerosis. Hum Pathol. 1993;24:477-82.

11. Schlatmann TJ, Becker AE. Pathogenesis of dissecting aortic aneurysm of the aorta. Comparative histopathologic study of significance of medial changes. Am J Cardiol. 1977;39:21-6.

12. Novaro GM, Tiong IY, Pearce GL, Grimm RA, Smedira N, Griffin BP. Features and predictors of ascending aortic dilatation in association with a congenital bicuspid aortic valve. Am J Cardiol. 2003;92:99-101.

13. Nistri S, Sorbo MD, Marin M, Palisi M, Scognamiglio R, Thiene G. Aortic root dilatation in young men with normally functioning bicuspid aortic valves. Heart. 1999;82:19-22.

14. Bonderman D, Gharehbaghi-Schnell E, Wollenek G, Maurer G, Baumgartner H, Lang IM. Mechanisms underlying aortic dilatation in congenital aortic valve malformation. Circulation. 1999;99:2138-43.

15. Matthias Bechtel JF, Noack F, Sayk F, Erasmi AW, Bartels C, Sievers $\mathrm{HH}$. Histopathological grading of ascending aortic aneurysm: comparison of patients with bicuspid versus tricuspid aortic valve. $J$ Heart Valve Dis. 2003;12:54-61.

16. Bauer M, Pasic M, Meyer R, Goetze N, Bauer U, Siniawski H, et al. Morphometric analysis of aortic media in patients with bicuspid and tricuspid aortic valve. Ann Thorac Surg. 2002;74:58-62.

17. Thyberg J, Hultgardh-Nilsson A. Fibronectin and the basement membrane components laminin and collagen type IV influence the pheno- typic properties of subcultured rat aortic smooth muscle cells differently. Cell Tissue Res. 1994;276:263-71.

18. Mackie EJ, Scott-Burden T, Hahn AW, Kern F, Bernhardt J, Regenass $\mathrm{S}$, et al. Expression of tenascin by vascular smooth muscle cells. Alterations in hypertensive rats and stimulation by angiotensin II. Am J Pathol. 1992;141:377-88.

19. Bardy N, Merval R, Benessiano J, Samuel JL, Tedgui A. Pressure and angiotensin II synergistically induce aortic fibronectin expression in organ culture model of rabbit aorta. Evidence for a pressure-induced tissue renin-angiotensin system. Circ Res. 1996;79:70-8.

20. Sho E, Sho M, Singh TM, Nanjo H, Komatsu M, Xu C, et al. Arterial enlargement in response to high flow requires early expression of matrix metalloproteinases to degrade extracellular matrix. Exp Mol Pathol. 2002;73:142-53.

21. Grande KJ, Cochran RP, Reinhall PG, Kunzelman KS. Stress variations in the human aortic root and valve: the role of anatomic asymmetry. Ann Biomed Eng. 1998;26:534-45.

22. Beller CJ, Labrosse MR, Thubrikar MJ, Robicsek F. Role of aortic root motion in the pathogenesis of aortic dissection. Circulation. 2004;109:763-9.

23. Hedin U, Holm J, Hansson GK. Induction of tenascin in rat arterial injury. Relationship to altered smooth muscle cell phenotype. Am J Pathol. 1991;139:649-56.

24. Sternik L, Zehr KJ, Schaff HV. A method of repair for asymmetric aneurysmal dilatation of the ascending aorta. Ann Thorac Surg. 2002;73:1332-4. 
TABLE E1. Demographics, aortic valve features, and aortic diameter in BAV patients

\begin{tabular}{|c|c|c|c|c|c|c|c|}
\hline Patient No. & Age (y) & Sex & BSA $\left(\mathrm{m}^{2}\right)$ & BAV disease & Degree & Ascending aorta (cm) & Cusp fusion pattern \\
\hline 1 & 48 & $\mathrm{M}$ & 2.00 & Regurgitation & $4+$ & 5.0 & Left/right \\
\hline 2 & 44 & $\mathrm{M}$ & 2.20 & Regurgitation & $4+$ & 6.5 & Left/right \\
\hline 3 & 60 & $\mathrm{~F}$ & 1.52 & Regurgitation & $4+$ & 4.5 & Right/noncoronary \\
\hline 4 & 57 & $\mathrm{M}$ & 1.70 & Regurgitation & $3+$ & 5.0 & Left/right \\
\hline 5 & 65 & $\mathrm{M}$ & 2.20 & Regurgitation & $4+$ & 6.0 & Not available* \\
\hline 6 & 66 & $\mathrm{M}$ & 1.67 & Regurgitation & $4+$ & 4.8 & Right/noncoronary \\
\hline 7 & 49 & $\mathrm{M}$ & 1.83 & Regurgitation & $4+$ & 5.1 & Left/right \\
\hline 8 & 50 & $\mathrm{~F}$ & 1.84 & Regurgitation & $4+$ & 4.9 & Left/right \\
\hline 9 & 63 & $\mathrm{M}$ & 1.82 & Regurgitation & $4+$ & 5.0 & Right/noncoronary \\
\hline 10 & 70 & $\mathrm{~F}$ & 1.82 & Regurgitation & $3+$ & 8.3 & Left/right \\
\hline 11 & 61 & $\mathrm{M}$ & 1.73 & Regurgitation & $4+$ & 6.2 & Not available* \\
\hline 12 & 67 & $\mathrm{M}$ & 1.66 & Regurgitation & $4+$ & 4.9 & Right/noncoronary \\
\hline 13 & 47 & $\mathrm{M}$ & 1.60 & Regurgitation & $4+$ & 4.7 & Left/right \\
\hline 14 & 41 & $\mathrm{M}$ & 1.94 & Regurgitation & $4+$ & 6.4 & Not available* \\
\hline 15 & 56 & $\mathrm{~F}$ & 2.10 & Regurgitation & $3+$ & 5.8 & Left/right \\
\hline 16 & 47 & $\mathrm{M}$ & 1.84 & Stenosis & $4+$ & 5.5 & Left/right \\
\hline 17 & 74 & $\mathrm{~F}$ & 1.83 & Stenosis & $4+$ & 4.6 & Right/noncoronary \\
\hline 18 & 60 & $\mathrm{M}$ & 1.82 & Stenosis & $4+$ & 4.7 & Right/noncoronary \\
\hline 19 & 75 & $\mathrm{M}$ & 1.71 & Stenosis & $4+$ & 5.0 & Not available* \\
\hline 20 & 45 & $\mathrm{~F}$ & 1.74 & Stenosis & $3+$ & 6.2 & Left/right \\
\hline 21 & 71 & $\mathrm{M}$ & 1.70 & Stenosis & $4+$ & 4.5 & Right/noncoronary \\
\hline 22 & 55 & $\mathrm{~F}$ & 1.90 & Stenosis & $4+$ & 5.7 & Left/right \\
\hline 23 & 58 & $\mathrm{~F}$ & 1.81 & Stenosis & $4+$ & 6.0 & Not available* \\
\hline 24 & 74 & $\mathrm{M}$ & 1.84 & Stenosis & $4+$ & 5.4 & Right/noncoronary \\
\hline 25 & 55 & $\mathrm{~F}$ & 1.77 & Stenosis & $3+$ & 6.5 & Left/right \\
\hline 26 & 47 & $\mathrm{M}$ & 1.86 & Stenosis & $4+$ & 7.4 & Right/noncoronary \\
\hline 27 & 43 & $\mathrm{M}$ & 1.96 & Stenosis & $4+$ & 7.5 & Not available* \\
\hline
\end{tabular}

$B A V$, Bicuspid aortic valve; $B S A$, body surface area. *Unidentified at echocardiography and not reported in the operative note.

APPENDIX E1. Oligonucleotide sequences used for RT-PCR

\begin{tabular}{|c|c|c|c|}
\hline Target mRNA & Primer & Sequence & PCR product (bp) \\
\hline \multirow[t]{2}{*}{ GAPDH } & Forward & 5'-GAGTCAACGGATTTGGTC-3' & \multirow[t]{2}{*}{238} \\
\hline & Reverse & 5'-TTGATTTTGGAGGGATCT-3' & \\
\hline \multirow[t]{2}{*}{ Fibronectin } & Forward & 5' -CCCAACTGGCATTGACTTTT-3' & \multirow[t]{2}{*}{136} \\
\hline & Reverse & 5' -CTCGAGGTCTTTCACTGAAG-3' & \\
\hline \multirow[t]{2}{*}{ Laminin $\alpha_{2}$} & Forward & 5'-AAAGTATCTGTGTCTTCAGGAGGTGC-3' & \multirow[t]{2}{*}{474} \\
\hline & Reverse & 5' -GCAGCCAGTGAATGTAATCACACGTC-3' & \\
\hline \multirow[t]{2}{*}{ Collagen I $\alpha_{1}$} & Forward & 5'-CTCCCCAGAAGACACAGGAA-3' & \multirow[t]{2}{*}{203} \\
\hline & Reverse & 5'-TCAAAAACGAAGGGGAGATG-3' & \\
\hline \multirow[t]{2}{*}{ Collagen III $\alpha_{1}$} & Forward & 5'-TACGGCAATCCTGAACTT-3' & \multirow[t]{2}{*}{245} \\
\hline & Reverse & 5'-GTGTGTTTCGTGCAACCA-3' & \\
\hline \multirow[t]{2}{*}{ Collagen IV $\alpha_{2}$} & Forward & 5'-CCAAGGAAGAGGTGGTGT-3' & \multirow[t]{2}{*}{169} \\
\hline & Reverse & 5'-GTGCTTCACCAGGAGGTA-3' & \\
\hline \multirow[t]{2}{*}{ Tenascin C } & Forward & 5' -TCAAGGCTGCTACGCCTTAT-3' & \multirow[t]{2}{*}{230} \\
\hline & Reverse & 5' -GTTCTGGGCTGCCTCTACTG-3' & \\
\hline
\end{tabular}

Primer sequences were designed by using the software Primer 3 (developed by Steve Rozen and Helen J. Skaletsky), which is available online at http://www-genome.wi.mit.edu. The optimal annealing temperature for all the primer pairs was $55^{\circ} \mathrm{C}$. The amplification of the GAPDH gene was used as a double internal control. The ratio between the samples and the housekeeping gene was calculated to normalize for initial variations in sample concentration and as a control for reaction efficiency. RT-PCR, Reverse transcription-polymerase chain reaction; mRNA, messenger RNA; GAPDH, glyceraldehyde phosphate dehydrogenase; $b p$, base pairs. 